\title{
Lipocalin 2 inversely regulates TRAIL sensitivity through p38 MAPK-mediated DR5 regulation in colorectal cancer
}

\author{
SE-LIM KIM ${ }^{1,2^{*}}$, IN SUK MIN ${ }^{1,2^{*}}$, YOUNG RAN PARK ${ }^{1,2}$, SOO TEIK LEE ${ }^{1,2}$ and SANG-WOOK KIM ${ }^{1,2}$ \\ ${ }^{1}$ Department of Internal Medicine and Rescearch Institute of Clinical Medicine, and ${ }^{2}$ Biomedical Research Institute, \\ Chonbuk National University Hospital, Chonbuk National University Medical School, Jeonju 561-712, Korea
}

Received July 3, 2018; Accepted August 28, 2018

DOI: 10.3892/ijo.2018.4562

\begin{abstract}
TNF-related apoptosis-inducing ligand (TRAIL) induces apoptosis through death receptors (DRs) 4 and/or 5 expressed on the cell surface. Multiple clinical trials are underway to evaluate the antitumor activity of recombinant human TRAIL and agonistic antibodies to DR4 or DR5. However, their therapeutic potential is limited by the high frequency of cancer resistance. In this study, we provide evidence demonstrating the role of lipocalin 2 (LCN2) in the TRAIL-mediated apoptosis of human colorectal cancer (CRC). By analyzing the mRNA expression data of $71 \mathrm{CRC}$ tissues from patients, we found that DR5 was preferentially expressed in CRC tissues with a low LCN2 expression level compared to tissues with a high LCN2 expression level. Moreover, we analyzed the association between DR5 and LCN2 expression and this analysis revealed that DR5 expression in CRC tended to be inversely associated with LCN2 expression. By contrast, no association was found between the DR4 and LCN2 expression levels. The expression patterns of LCN2 in human CRC cell lines also exhibited an inverse association with DR5 expression. The knockdown of LCN2 by siRNA in the TRAIL-resistant CRC cells expressing high levels of LCN2 led to a significant increase in TRAIL-induced apoptosis through the upregulation of DR5 protein and mRNA expression. The mechanism through which LCN2 silencing sensitized the CRC cells to TRAIL was dependent on the extrinsic pathway of apoptosis. In addition, we identified that the knockdown of LCN2 enhanced the sensitivity of the cells to TRAIL through the p38 MAPK/CHOP-dependent upregulation of DR5. Taken
\end{abstract}

Correspondence to: Dr Sang-Wook Kim, Department of Internal Medicine and Research Institute of Clinical Medicine, Chonbuk National University Hospital, Chonbuk National University Medical School, Jeonju 561-712, Korea

E-mail: clickm@jbnu.ac.kr

*Contributed equally

Key words: lipocalin 2, tumor necrosis factor-related apoptosisinducing ligand, resistance, death receptor, apoptosis, mitogen-activated protein kinase, colorectal cancer together, the findings of this study suggest that LCN2 is responsible for TRAIL sensitivity and LCN2 may thus prove to be a promising target protein in DR-targeted CRC therapy.

\section{Introduction}

Colorectal cancer (CRC) is one of the most commonly diagnosed malignancies and is a leading cause of cancer-related mortality worldwide $(1,2)$. Although CRC diagnosis and treatment strategies have improved over the past decades, CRC is still considered a major public health concern (3). Despite the development of novel target agents for CRC therapy, the survival rate still remains unsatisfactory $(4,5)$.

Tumor necrosis factor (TNF)-related apoptosis-inducing ligand (TRAIL) is a member of the tumor necrosis factor family of cytokines. Due to their cancer cell specificity and marked anticancer effects in a variety of pre-clinical trials, TRAIL is considered an attractive anticancer agent (6). TRAILinduced apoptosis is mediated by binding to cell surface death receptor (DR)5 (also known TRAIL-R2) or DR4 (also known TRAIL-R1) (7). The therapeutic potential of soluble TRAIL (sTRAIL) and of monoclonal antibodies directed against DRs have been extensively exploited in phase I/II clinical trials (8-10). However, despite promising preclinical data and favorable toxicity profiles, these attempts have displayed poor anticancer activity and limitations in use due to the frequent incidence of primary or acquired tumor cell resistance, which can occur in CRC (6). Among strategies which can be used to overcome TRAIL resistance, combined treatment is an attractive method that enhances TRAIL sensitivity. In our previous study, we demonstrated that the potent NF- $\mathrm{B}$ inhibitor, parthenolide, sensitized resistant CRC cells to TRAIL, and combined treatment with parthenolide represented a possible novel therapeutic strategy for CRC treatment (11). For better clinical outcomes, an in-depth analysis of the resistance mechanisms and the identification of markers to predict response to TRAIL are warranted.

Lipocalin 2 (LCN2), also known as oncogene 24p3 neutrophil gelatinase-associated lipocalin (NGAL), siderocalin, or uterocalin, is a $25 \mathrm{kDa}$ secreted glycoprotein that belongs to the lipocalin superfamily (12). LCN2 is an effective carrier of small, hydrophobic ligands and exerts bacteriostatic effects by sequestering the siderophore-iron complex $(13,14)$. In addition to these immune defense functions, LNC2 is involved in acute 
phase response, kidney cell differentiation, erythropoiesis and iron metabolism $(13,15-17)$. Furthermore, LCN2 is involved in cancer biology. LCN2 has been identified in colonic epithelial cells under various inflammatory conditions, inflammatory bowel diseases and colorectal cancers (15). In vitro experimental evidence has indicated the inverse association between LCN2 and cell motility, and LCN2 has been shown to suppress CRC progression and metastasis (18). Our previous study demonstrated that LCN2 was negatively associated with an advanced stage and metastasis (19). Thus, LCN2 may play a differential role in early and late stage disease. However, a deep understanding of the role of LCN2 as a molecular target is lacking. Moreover, if a new perspective of LCN2 as a target for chemoresistance is suggested, this may provide new insight into the role of LCN2 in cancer.

The present study was undertaken to investigate the potential of LCN2 as a target molecule for predicting TRAIL sensitivity and to explore the molecular mechanisms that regulate TRAIL resistance by LCN2. Our findings may provide new insight into TRAIL resistance in CRC and may lead to the development of novel therapeutic targets for the disease.

\section{Materials and methods}

Chemicals and reagents. TRAIL, which was purchased from Pepprotech (Rocky Hill, NJ, USA), was dissolved in 1X PBS to a concentration of $100 \mathrm{ng} / \mu \mathrm{l}$. Annexin V-FITC and propidium iodide (PI) were purchased from Thermo Fisher Scientific (Waltham, MA, USA). The caspase inhibitor, z-VAD-FMK, and the mitogen-activated protein kinase (MAPK) inhibitor, SB203580, were obtained from Sigma-Aldrich (St. Louis, MO, USA). The protein levels of DR4 and DR5 were analyzed using phycoerythrin (PE)-conjugated DR4 and DR5 obtained from ProSci Inc. (San Diego, CA, USA).

Patients and tissue specimens. A total of $71 \mathrm{CRC}$ tissues were obtained through the Biobank of Chonbuk National University Hospital, a member of the National Biobank of Korea. All patients had a pathological diagnosis of CRC. The samples were frozen in liquid nitrogen and stored at $-80^{\circ} \mathrm{C}$. This study consisted of $28(39.4 \%)$ females and $43(60.5 \%)$ males with a mean age of 63.1 years. All patients provided written informed consent prior to collecting the tissue samples. The study protocol was approved by the Institutional Review Boards of Chonbuk National University Hospital (IRB no. 2016-04-018).

RNA isolation and reverse transcription-quantitative polymerase chain reaction $(R T-q P C R)$. Total RNA was extracted from the cells and human normal tissue/matched tumor samples using TRIzol reagent (Thermo Fisher Scientific). Reverse transcription was performed using M-MLV Reverse Transcriptase (Promega, Madison, WI, USA) according to the manufacturer's instructions. qPCR was performed using an ABI 7500 real-time PCR system (Applied Biosystems, Foster City, CA, USA). In brief, $20 \mu \mathrm{l}$ of master mix were prepared on ice with $10 \mu \mathrm{l}$ of $2 \mathrm{X}$ SYBR-Green, $1 \mu \mathrm{l}$ of primers, $2 \mu \mathrm{l}$ of DNA and $7 \mu \mathrm{l}$ of nuclease-free water. The master mix was initially denatured at $95^{\circ} \mathrm{C}$ for $10 \mathrm{~min}$ followed by 40 cycles of denaturation at $95^{\circ} \mathrm{C}$ for $15 \mathrm{sec}$, annealing and extension at $60^{\circ} \mathrm{C}$ for $30 \mathrm{sec}$. The geometric average $\mathrm{Cq}$ value was used to calculate the relative expression of LCN2 using the $2^{-\Delta \Delta C q}$ method, which was normalized to beta-2-microglobulin (B2M) (20). The primers used in this experiment were as follows: 5'-TCACCTCCGTCCTGTTTAGG-3' (forward) and 5'-CGAAGTCAGCTCCTTGGTTC-3' (reverse) for LCN2; '5-CACAGCAATGGGAACATAGC-3' (forward) an '5-CAG GGACTTCTCTCTTCTTC-3' (reverse) for DR4; 5'-CTGAAA GGCATCTGCTCAGGTG-3' (forward) and 5'-CAGAGTCT GCA TTAC CTTCTAG-3' (reverse) for DR5; 5'-CCTGAATTG CTATGTGTCTGGG-3' (forward) and 5'-TGATGCTGCTT ACATGTCTCGA-3' (reverse) for B2M. The LCN2 expression level was subdivided into he low and high groups according to the mean value.

Cell culture. The human colorectal cancer cell lines, HT-29, DLD-1, SW480, HCT116 and SW620, were purchased from the American Type Culture Collection (ATCC, Manassas, VA, USA). The cells were cultured in RPMI-1640 medium supplemented with $10 \%$ fetal bovine serum (FBS), 100 units penicillin and 100 units streptomycin in a humidified $5 \% \mathrm{CO}_{2}$ atmosphere at $37^{\circ} \mathrm{C}$.

Small interfering RNA (siRNA) for the inhibition of gene expression. The siRNA sequences used for the targeted silencing of the LCN2 gene (NCBI Ref Seq NM_005564.4) were from Ambion (Austin, TX, USA), and those for the DR5 gene (NCBI Ref Seq NM_003842) were from Santa Cruz Biotechnology (Santa Cruz, CA, USA). LCN2 siRNA, DR5 siRNA and scrambled siRNA as the negative control (Ambion) were transfected into the HT-29 and DLD-1 cells using TransiT-X $2^{\circledR}$ transfection reagent (Mirus Bio, Madison, WI, USA) according to the manufacturer's instructions.

Reverse transcription-PCR (RT-PCR). Total RNA was isolated from the cultured cells using TRIzol reagent (Invitrogen/ Thermo Fisher Scientific), and cDNA was synthesized with Super Script II reverse-transcriptase (Invitrogen/Thermo Fisher Scientific) according to the manufacturer's instructions. GAPDH expression was used as an internal control. The following primer sequences were used: 5'-TCACCTCCGTCC TGTTTAGG-3' (forward) and 5'-CGAAGTCAGCTCCTTGG TTC-3' (reverse) for LCN2, and 5'-CAGGTGTCAACATGTT GTCC-3' (forward) and 5'-ATCGAAGCACTGTCTCAGAG-3' (reverse) for DR5, which generate 242 and 136 bp products, respectively. Following initial denaturation at $95^{\circ} \mathrm{C}$ for $1 \mathrm{~min}$, PCR was performed for various cycles $\left(30 \mathrm{sec}\right.$ at $94^{\circ} \mathrm{C}, 1 \mathrm{~min}$ at annealing temperature and $2 \mathrm{~min}$ at $72^{\circ} \mathrm{C}$ ) using Taq polymerase. The reaction products $(10 \mu \mathrm{l})$ were separated on a $2 \%$ agarose gel and stained with Redsafe ${ }^{\mathrm{TM}}$ (Intron, Daejeon, Korea). The DNA band intensity was analyzed by densitometry using an $\mathrm{N} \alpha \mathrm{BI}$ imager (Neogene Science, Suwon, Korea).

Quantification of DR expression on the cell surface. To quantify the cell surface expression of DR4 and DR5, the cells were harvested by trypsinization, washed in PBS, and incubated for $30 \mathrm{~min}$ at $4^{\circ} \mathrm{C}$ with $500 \mathrm{ng} /$ test of phycoerythrin (PE)-conjugated monoclonal anti-human DR4 (cat. no. 12-6644-41) and DR5 (cat. no. 12-9908-42) antibodies (eBiosciences, San Diego, CA, USA). Non-immune mouse IgG was used as the negative control. Fluorescence was measured 
using a BD LSR flow cytometer and processed with CellQuest software for analysis.

Cell viability assay. The DLD-1 and HT-29 cells were plated at a density of $1.0 \times 10^{4}$ cells per well in 96-well plates. Following transfection with scrambled siRNA or LCN2 siRNA, the medium was removed, and $200 \mu \mathrm{l}$ of fresh medium, including various concentrations of TRAIL $(10,50,100,300$ and $500 \mathrm{ng} / \mathrm{ml}$ ) were added. Subsequently, $20 \mu \mathrm{l}$ of 3-(4,5-dimethylthiazol-2yl)-2,5-diphenyltetrazolium bromide (MTT, $2.5 \mathrm{mg}$ dissolved in $50 \mu \mathrm{l}$ of DMSO; Sigma-Aldrich) were added to each well. Following incubation for $4 \mathrm{~h}$ at $37^{\circ} \mathrm{C}$, the culture medium containing MTT was removed and $200 \mu \mathrm{l}$ of DMSO were added. This was followed by shaking until the crystals were dissolved. Viable cells were detected by measuring the absorbance at $570 \mathrm{~nm}$ using a microplate reader (Molecular Devices, Sunnyvale, CA, USA).

Detection of apoptosis. Apoptotic cell death was determined by staining the cells with Annexin V-FITC (Ex/Em, 488/519 $\mathrm{nm})$. In brief, $1 \times 10^{5}$ cells in a $60-\mathrm{mm}$ culture dish were transfected with scrambled siRNA or LCN2 siRNA. After $48 \mathrm{~h}$, the cells were pre-treated with Z-VAD-FMK (10 nM) or SB203580 $(50 \mu \mathrm{M})$ for $1 \mathrm{~h}$, and $50 \mathrm{ng} / \mathrm{ml}$ of TRAIL was then added to the transfected cells for $24 \mathrm{~h}$. The cells were washed twice with cold PBS and then resuspended in $500 \mu \mathrm{l}$ of binding buffer (10 mM HEPES/NaOH pH 7.4, $140 \mathrm{mM} \mathrm{NaCl}$, and $2.5 \mathrm{mM}$ $\mathrm{CaCl}_{2}$ ) at a concentration of $1 \times 10^{6}$ cells $/ \mathrm{ml}$. Annexin V-FITC (5 $\mu \mathrm{l})$ and PI $(1 \mu \mathrm{g} / \mathrm{ml})$ was then added, and the cells were analyzed with a BD FACSCalibur ${ }^{\mathrm{TM}}$ (BD Biosciences, San Jose, CA, USA).

Protein extraction and western blot analysis. The DLD-1 and HT-29 cells were harvested by resolving in RIPA buffer $(50 \mathrm{mM}$ Tris- $\mathrm{HCl}, 150 \mathrm{mM} \mathrm{NaCl}, 1 \%$ Triton $\mathrm{X}-100$, $1 \%$ sodium deoxycholate, $0.1 \%$ SDS and protease inhibitors) and were centrifuged at $1,3870 \times \mathrm{g}$ at $4^{\circ} \mathrm{C}$ for $30 \mathrm{~min}$. Following centrifugation, supernatants were used as whole cell extracts. The protein concentration in the cell lysates or tissue lysates was measured using a Protein Quantification kit from Bio-Rad (Hercules, CA, USA). Subsequently, $50 \mu \mathrm{g}$ of protein or $30 \mu \mathrm{g}$ of protein per lane were loaded onto $8-15 \%$ SDS-polyacrylamide gels. After transferring and blocking using $3 \%$ of bovine albumin serum, the polyvinylidene difluoride (PVDF) membranes were probed with various antibodies [anti-LCN2 (1:1,000; AF1757; R\&D Systems,Minneapolis, MN, USA), anti-DR4 (1:1,000; \#1167; ProSci $\Psi^{\mathrm{TM}}$, Poway, CA, USA), anti-DR5 (1:1,000; \#2019; ProSci $\left.\Psi^{\text {TM }}\right)$, anti-Fasassociated death-domain-like IL-1 $\beta$-converting enzyme (FLICE)-inhibitory protein (FLIP, 1:1,000; \#8510; Cell Signaling Technology, Danvers, MA, USA), anti-Bid (1:1,000; SC-11423; Santa Cruz Biotechnology), anti-caspase-8 (1:1,000; SC-73526; Santa Cruz Biotechnology), anti-caspase-3 (1:1,000; SC-7148; Santa Cruz Biotechnology), anti-cleaved caspase-3 (1:1,000; \#9661; Cell Signaling Technology), anti-poly(ADP-ribose) polymerase (PARP, 1:1,000, SC-7150; Santa Cruz Biotechnology), anti-Bcl-xL (1:1,000; SC-8392; Santa Cruz Biotechnology), anti-Bcl-2 (1:1,000; SC-783; Santa Cruz Biotechnology), anti-cytochrome $c$ (1:1,000, SC-65396; Santa Cruz Biotechnology), anti-caspase-9 (1:1,000; \#9502S;
Cell Signaling Technology), anti-phospho-ERK (1:1,000; \#9106; Cell Signaling Technology), anti-phospho-p38 (1:1,000, \#4511; Cell Signaling Technology), anti-phosphoJNK (1:1,000; \#4668; Cell Signaling Technology), anti-C/EBP homologous protein (CHOP, 1:1,000; \#2895; Cell Signaling Technology) and anti-actin (1:2,000; A2066; Sigma-Aldrich) antibodies] in $4^{\circ} \mathrm{C}$ for overnight. HRP-conjugated goat anti-rabbit IgG (SC-2004; Santa Cruz Biotechnology), goat anti-mouse (SC-2005; Santa Cruz Biotechnology) and mouse anti-goat (SC-2354; Santa Cruz Biotechnology) secondary antibodies were used at a concentration of 1:3,000 for $1 \mathrm{~h}$ at room temperature. Binding of the antibody to the antigen was detected using enhanced ECL prime (GE Healthcare, NJ, USA) and was captured and analyzed by the Las-3000 luminescent Image Analyzer (Fuji Film, Tokyo, Japan).

Statistical analysis. The association between the LCN2 and DR4/5 levels in the human specimens was analyzed using the Chi-square $\left(\chi^{2}\right)$ test. The Student's t-test (for differences between 2 groups) or one-way analysis of variance (ANOVA) with Tukey's test were used to analyze differences between more than 2 groups. The data are presented as the means \pm SD of at least 3 independent experiments. All data were entered into Microsoft Excel 5.0, and GraphPad Prism 5.0 was used. A probability $(\mathrm{P})$-value $<0.05$ was considered to indicate a statistically significant difference.

\section{Results}

LCN2 and DR5 have a negative association in human CRC tissues and cells. To identify the association between LCN2 and DRs in patients with CRC, the expression levels of LCN2, DR4 and DR5 in the specimens from patients with CRC and CRC cell lines were examined. First, the mRNA levels of LCN2, DR4 and DR5 in 71 frozen tissues of patients with CRC was measured by RT-qPCR, and the association of the mRNA expression of each of the DRs with mRNA expression of LCN2 was analyzed. As shown in Fig. 1A, we did not observe any association between the DR4 and LCN2 mRNA levels in the CRC tissues. Of note, the LCN2 level was found to be negatively associated with the DR5 expression level in the CRC tissue samples. Of the 71 patients with CRC, the DR5 level in the LCN2 low expression group was 0.558-15.380 $(3.015 \pm 2.57)$ and the DR5 level in the LCN2 high expression group was 0.529-5.679 (1.823 \pm 1.168$)$. Moreover, our analysis indicated that LCN2 expression was negatively regulated along with DR5 expression in the CRC specimens (Fig. 1B).

Subsequently, western blot analysis was performed to determine the protein expression levels of DR4, DR5 and LCN2 in 5 CRC cell lines (HT-29, DLD-1 SW480, SW620 and HCT116) (Fig. 1C). The protein levels of LCN2 were higher in the HT-29 and DLD-1 cell lines than in the SW480, HCT116 and SW620 cell lines. However, the protein levels of DR5 were higher in the SW480, HCT116 and SW620 cell lines than in the HT-29 and DLD-1 cell lines. Conversely, all the cell lines exhibited similar levels of DR4. Taken together, these findings suggest that LCN2 is negatively associated with DR5 in CRC.

Downregulation of LCN2 enhances DR5 expression and sensitizes the cells to TRAIL-induced apoptosis. To define 
A
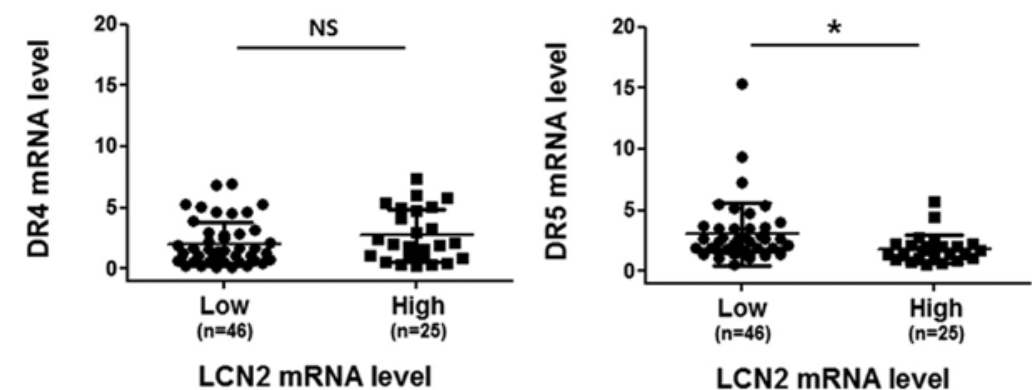

LCN2 mRNA level
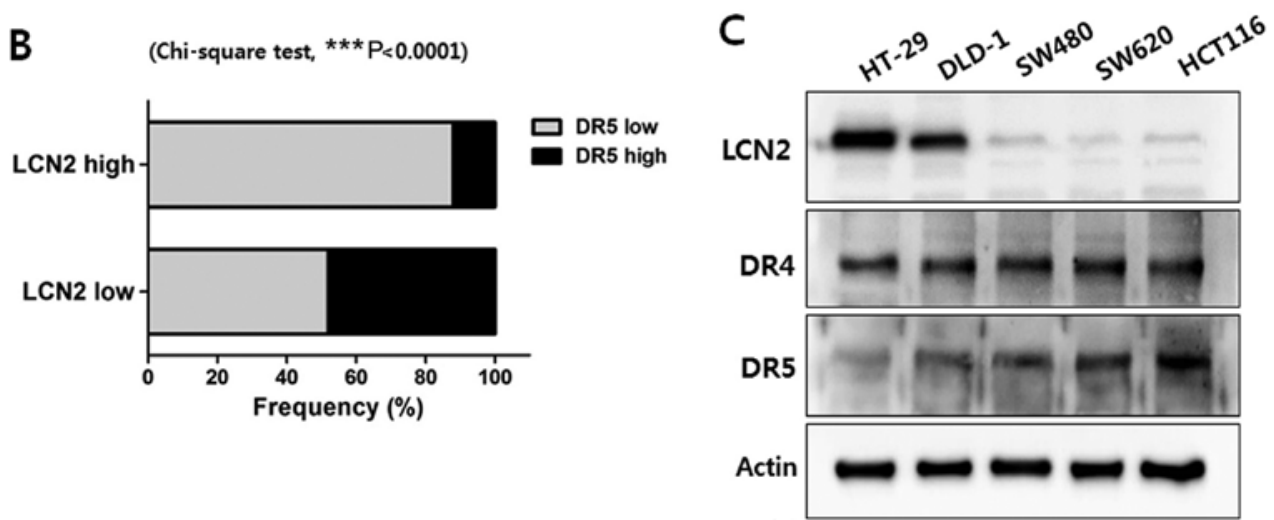

Figure 1. LCN2 and DR5 exhibit a negative association in CRC tissues from patients and CRC cells. (A) The mRNA levels of DR4/5 and LCN2 were determined by RT-qPCR in 71 CRC tissues. The samples were then divided into 2 groups according to the level of DR5 expression (high or low) and according to mean value of LCN2, LCN2-low or LCN2-high. (B) The association between LCN2 and DR5 in human CRC specimens was represented by the percentage of cases according to the classification of the mean value of LCN2 and DR5. (C) Protein level of DR4/5 and LCN2 determined by western blot analysis in human CRC cell lines. ${ }^{*} \mathrm{P}<0.05$ and ${ }^{* * * *} \mathrm{P}<0.0001$. LCN2, lipocalin 2; DR, death receptor; CRC, colorectal cancer.

the role of LCN2 in the regulation of DR5, the DLD-1/ HT-29 and HCT116 cells were employed as representatives of DR5-deficient cells and DR5-enriched cells, respectively. As shown in Fig. 2A, the DR5 protein and mRNA levels were increased by the silencing of LCN2 in the DLD-1/HT-29 cells. On the contrary, the DR4 protein level was not affected by transfection with LCN2 siRNA. Of note, the knockdown of DR5 in the HCT116 cells did not affect the regulation of LCN2 expression (Fig. 2B). These results were confirmed by flow cytometry. The expression of DR4 on the cell surface was not altered by transfection with LCN2 siRNA compared to transfection with scrambled siRNA; however, DR5 expression was markedly increased by the silencing of LCN2 in the DLD-1 and HT-29 cells (Fig. 2C). These results indicate that the inhibition of LCN2 promotes the expression of DR5, and that LCN2 regulates DR5 as an upstream factor from DR5 in TRAIL-induced apoptosis.

We also identified whether DR5 upregulation by LCN2 silencing affects the TRAIL-induced apoptosis of DR5-deficient cells. Cell viability assay was performed following treatment with various concentrations of TRAIL (50, 100,300 and $500 \mathrm{ng} / \mathrm{ml}$ ) for $24 \mathrm{~h}$ followed by transfection with scrambled siRNA or LCN2 siRNA. As shown by the results of MTT assay, TRAIL-induced cell death was increased by the silencing of LCN2 compared to the cells transfected with scrambled siRNA in both cell lines (Fig. 3A).

To ascertain the above-mentioned observations, apoptotic cell death analysis was performed using Annexin V/PI staining. As shown in Fig. 3B, the cells transfected with LCN2 siRNA exhibited greater apoptotic cell death when treated with $50 \mathrm{ng} / \mathrm{ml}$ of TRAIL compared to the cells transfected with scrambled siRNA. Moreover, the quantification of apoptotic cell death was carried out by counting cells in the lower right and upper right quadrants. The results revealed that TRAIL-induced apoptosis was significantly increased by the silencing of LCN2 from 22.8 to $49.2 \%$ in the DLD-1 cells and from 14.6 to $32.5 \%$ in the HT-29 cells. These results indicate that the downregulation of LCN2 enhances DR5 expression and promotes the TRAIL-induced apoptosis of TRAIL-resistant CRC cells.

Downregulation of LCN2 enhances TRAIL-mediated apoptotic signaling by engaging the extrinsic pathway. Having shown LCN2-related sensitization to TRAIL-induced apoptosis, we then investigated the regulation of the molecules involved in death signaling by western blot analysis (Fig. 4A). FLIPs are anti-apoptotic proteins that can be recruited to the death-inducing signaling complex (DISC) (21). As FLIP contains two death effector domains (DED) and an inactive caspase domain, FLIP can inhibit death receptor-mediated apoptosis by binding to Fas-associated death domain (FADD) or caspase-8 $(21,22)$. As shown in Fig. 4A, lower levels of FLIP were observed following treatment with TRAIL in the cells in which LCN2 was silenced (LCN2 siRNA + 50 ng/ ml TRAIL) compared to the control cells (scrambled siRNA $+50 \mathrm{ng} / \mathrm{ml}$ TRAIL). These results suggest that LCN2 
A
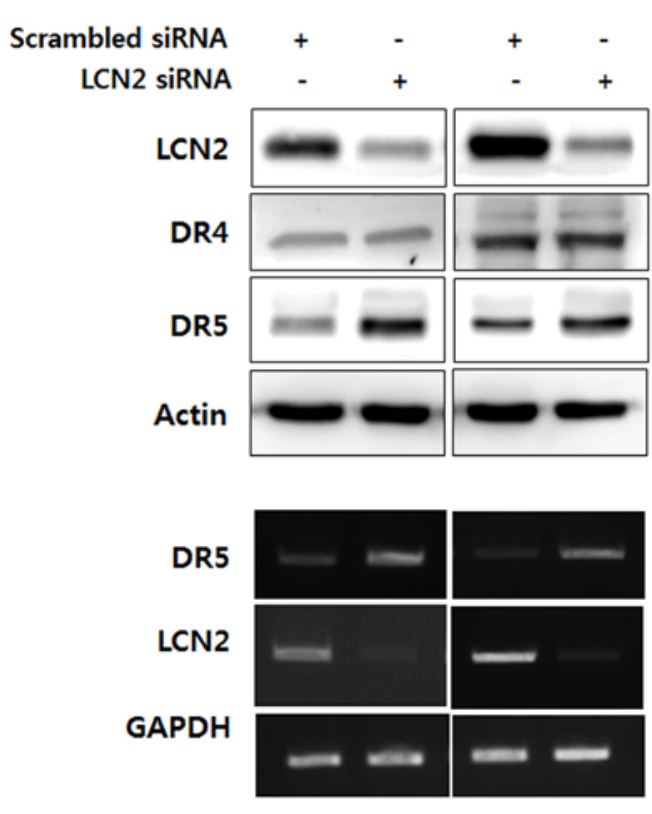

C
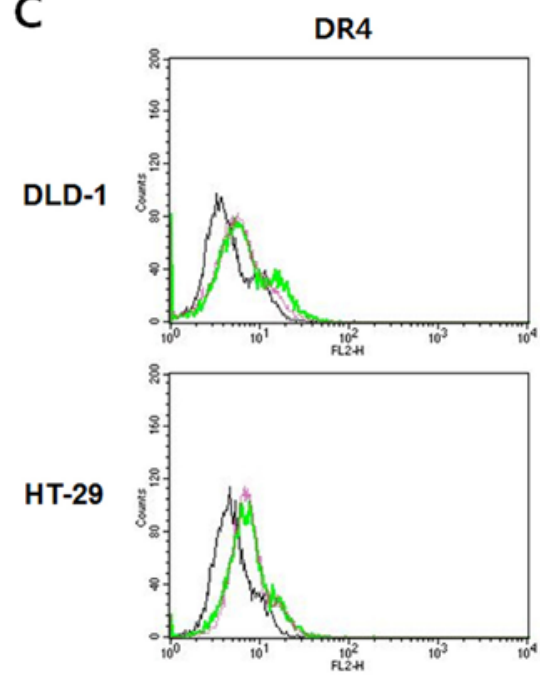

B

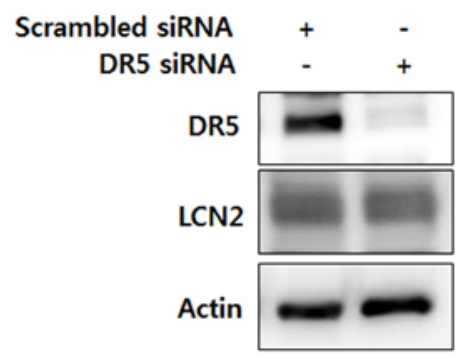

HCT116

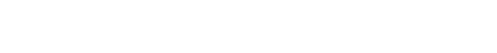
characteristics, were transfected with LCN2 siRNA and the endogenous levels of DR4/5 and LCN2 were then detected by western blot analysis and RT-PCR. (B) HCT116 cells with TRAIL sensitive characteristics were transfected with DR5 siRNA. LCN2 and DR5 levels were also evaluated by western blot analysis. (C) The cell surface expression of DR4 and DR5 was determined by flow cytometry using PE-conjugated DR4/5 antibody. In the histograms the live cell populations are presented as follows: Left panels (DR4): black line, scramble siRNA + IgG antibody; green line, scramble siRNA + DR4 antibody; pink line, LNC2 siRNA + DR4 antibody. Right panels (DR5): black line, scramble siRNA + IgG antibody; green line, scramble siRNA + DR5 antibody; pink line, LNC2 siRNA + DR5 antibody. LCN2, lipocalin 2; DR, death receptor; TRAIL, TNF-related apoptosis-inducing ligand.

affects FLIP to inhibit death receptor-mediated cell death (Fig. 4A, 2nd panel from the top). Thus, the decreased FLIP expression by treatment with TRAIL following the silencing of LCN2 can induce the processing of caspase- 8 activation. As expected, the cleavage of caspase- 8 was much higher in the TRAIL-treated cells in which LCN2 was silenced than in the TRAIL-treated cells transfected with the scrambled siRNA (Fig. 4A, 3rd panel from the top). To identify the next caspase cascade, the expression level of caspase- 3 was analyzed using full-length caspase- 3 and cleaved caspase- 3 antibody, respectively. During the activation of caspase- 3 , the cleaved form of caspase- 3 increases, while the full length decreases. As shown in the 4th and 5th panels (from the top) in Fig. 4A, caspase-3 activation was markedly induced in the TRAIL-treated cells in which LCN2 was silenced. These results indicate that the silencing of LCN2 enhances the TRAIL-induced cleavage of caspase- 8 and caspase- 3 in their active forms in TRAILresistant CRC cells. Following the activation of caspases during apoptosis, the cleavage of PARP occurs by several caspases, including caspase-3, which cleaves 113-kDa PARP into 89 - and $24-\mathrm{kDa}$ polypeptides $(23,24)$. Thus, in this study, to confirm the effects of LCN2 regulation on PARP cleavage, the cells were treated with $50 \mathrm{ng} / \mathrm{ml}$ of TRAIL following transfection with scrambled or LCN2 siRNA. As shown in the 6th panel (from the top) in Fig. 4A, a higher level of cleaved PARP following treatment with TRAIL was observed 
A

DLD-1

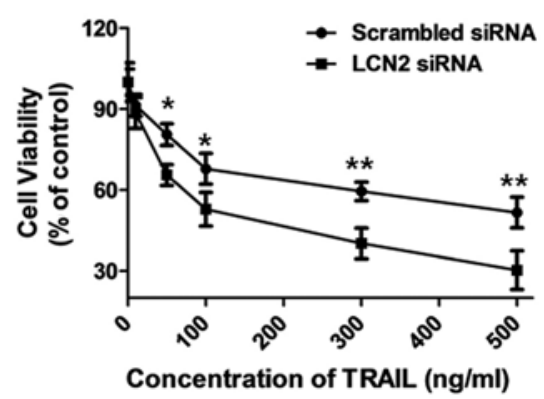

HT-29

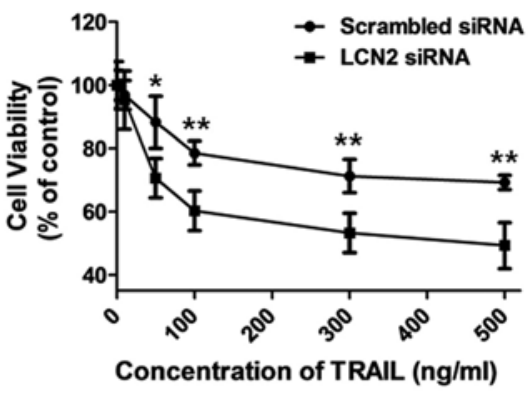

B
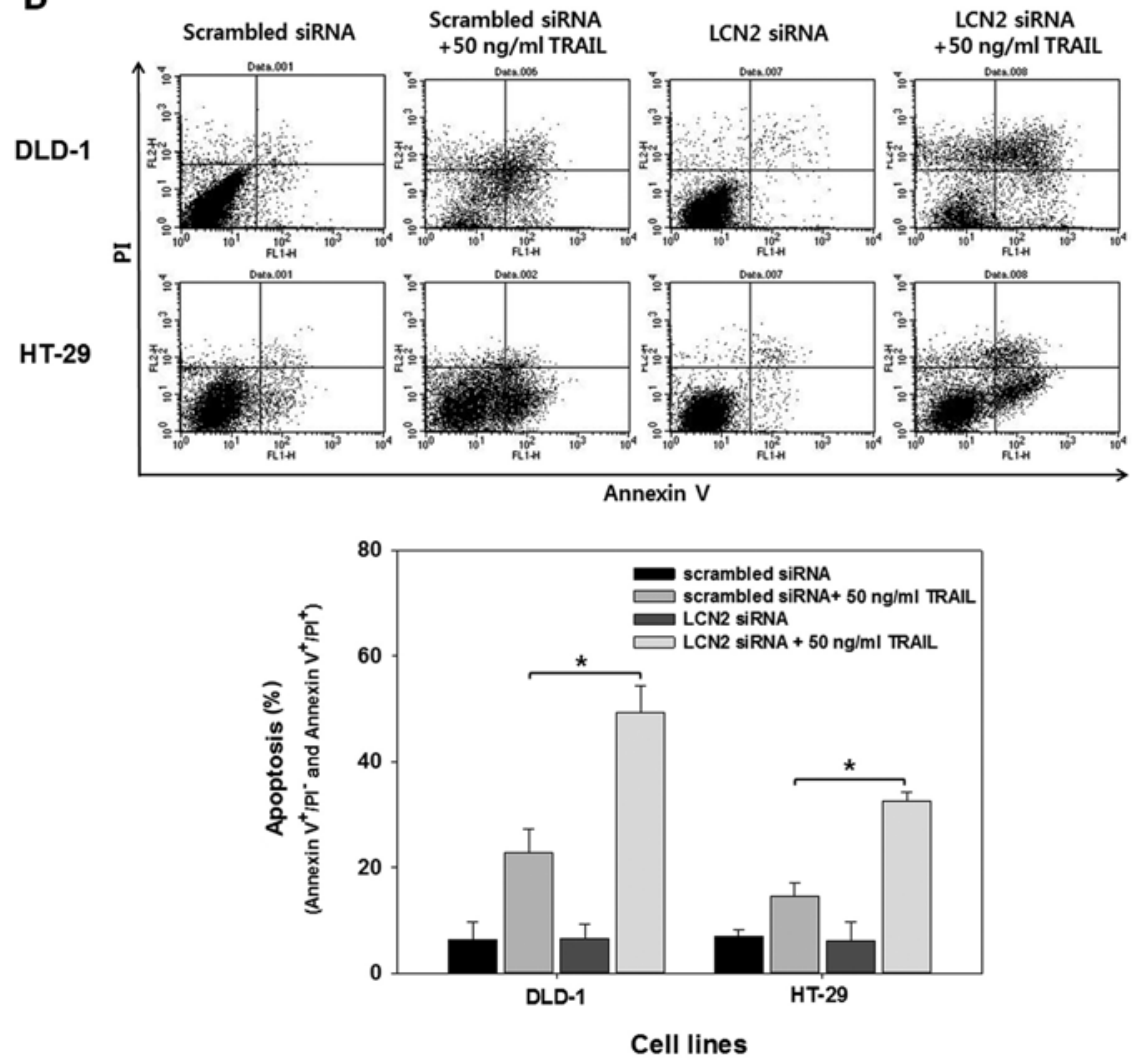

Figure 3. Knockdown of LCN2 sensitizes TRAIL-resistant cells to TRAIL-induced apoptosis. (A) Scrambled siRNA- or LCN2 siRNA-transfected cells were treated with various concentrations of TRAIL for $24 \mathrm{~h}$. The cells were then analyzed for viability by MTT assay. (B) Following transient transfection with scrambled or LCN2 siRNA, cells were stained with Annexin V/PI and analyzed using flow cytometry (upper panel) and the quantification of apoptosis (bottom panel). ${ }^{*} \mathrm{P}<0.05$ and ${ }^{* *} \mathrm{P}<0.001$. LCN2, lipocalin 2; TRAIL, TNF-related apoptosis-inducing ligand.

in the cells in which LCN2 was silenced compared with the scrambled siRNA-transfected cells treated with TRAIL.

To determine whether the TRAIL-induced apoptosis resulting from LCN2 silencing is dependent on the initiation of the extrinsic or intrinsic pathways, molecules involved in the intrinsic pathway were examined by western blot analysis (Fig. 4B). The expression of Bid, which links the extrinsic and intrinsic pathways, was not altered by LCN2 regulation with or without TRAIL treatment. Moreover, mitochondria-related anti-(Bcl-2 and Bcl-xL) or pro-apoptotic (cytochrome $c$ and caspase-9) molecules also exhibited similar levels with or without LCN2 regulation. Collectively, these data provide evidence that the downregulation of LCN2 enhances TRAIL-induced apoptosis through the extrinsic apoptotic pathway.
To further evaluate the findings shown in Fig. 4A, the cells in which LCN2 was silenced were pre-treated with Z-VAD-FMK, a general caspase inhibitor, and apoptotic cell death was evaluated by Annexin V assay. As shown in Fig. 4C, the apoptotic cell death induced by TRAIL treatment of the cells in which LCN2 was silenced decreased following pretreatment with Z-VAD-FMK. These results suggest that the downregulation of LCN2 sensitizes TRAIL-resistant CRC cells to TRAIL and induces TRAIL-mediated apoptosis related to the extrinsic pathway.

p38 MAPK and CHOP are involved in LCN2-related TRAIL sensitivity. Several studies have demonstrated that the regulation of DR5 and TRAIL sensitivity is mediated by MAPK signaling (25-28). Thus, in this study, to investigate the 
A

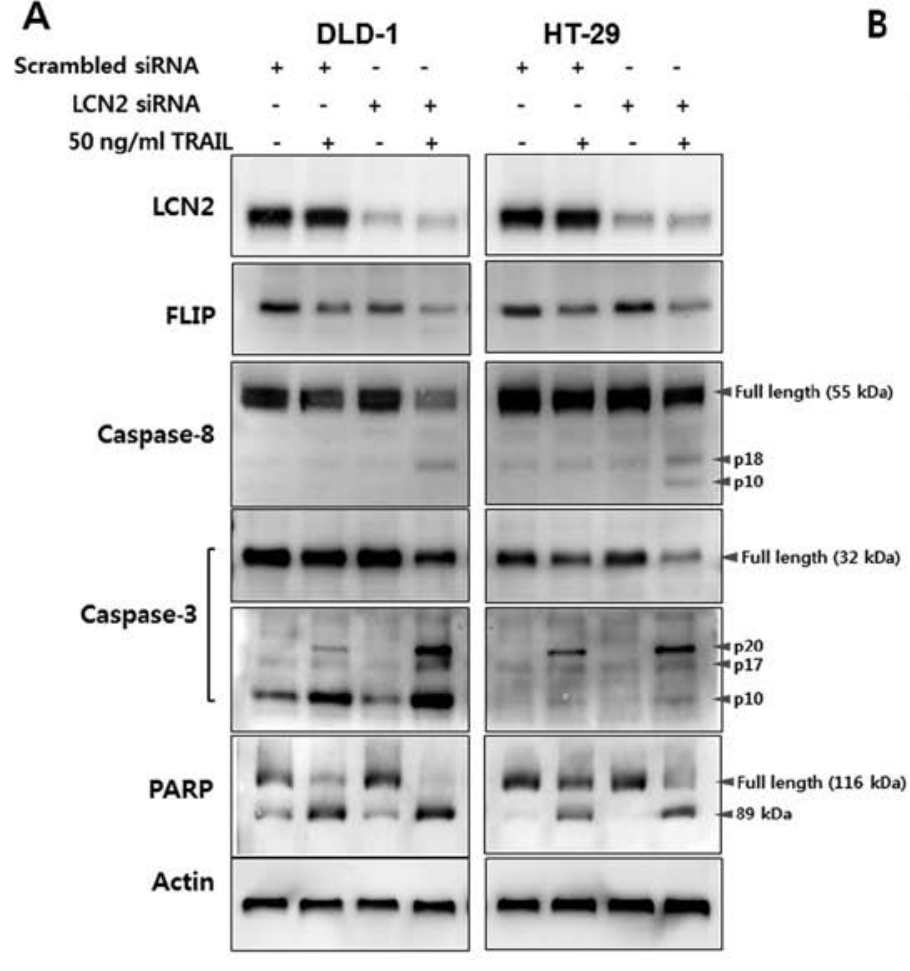

B

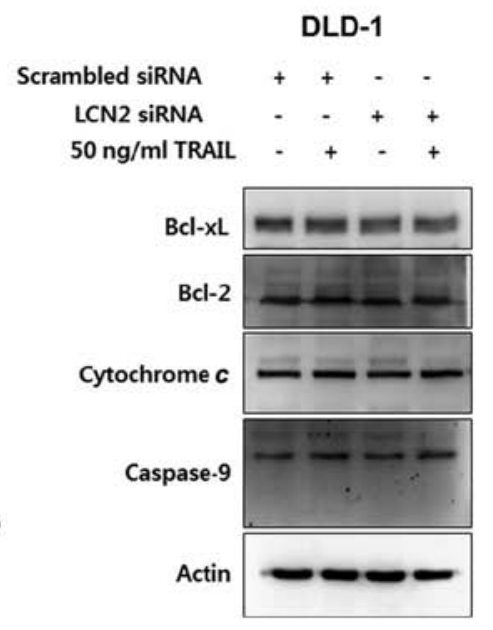

C

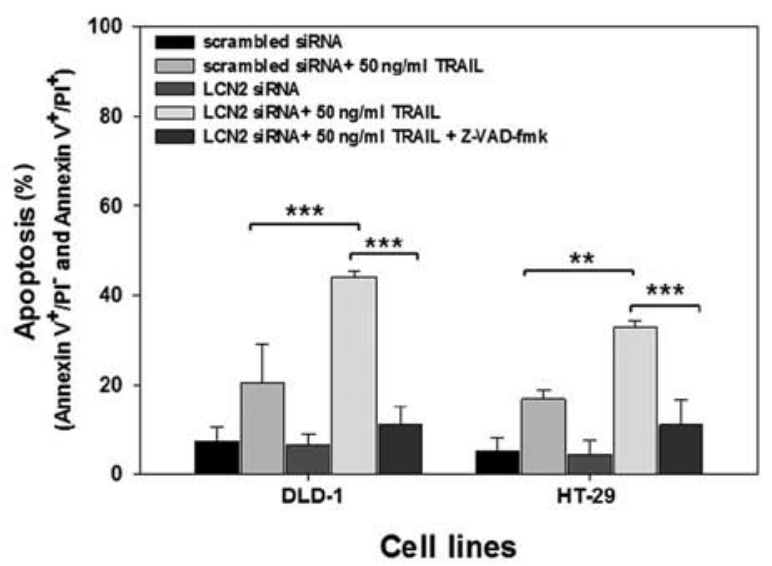

Figure 4. LCN2 knockdown by siRNA leads to TRAIL-induced apoptosis through the extrinsic pathway. (A) Scrambled siRNA- or LCN2 siRNA-transfected cells were treated with $50 \mathrm{ng} / \mathrm{ml}$ TRAIL for $24 \mathrm{~h}$. The regulation of apoptotic molecules related to the extrinsic apoptotic pathway was then examined by western blot analysis. (B) Transfected DLD-1 cells were treated with $50 \mathrm{ng} / \mathrm{ml}$ of TRAIL for $24 \mathrm{~h}$. The levels of proteins belonging to the intrinsic pathway (Bid, Bcl-xL, Bcl-2, cytochrome $c$ and caspase-9) were examined by western blot analysis. (C) Transfected cells were treated with $50 \mathrm{ng} / \mathrm{ml}$ of TRAIL in the presence of the general caspase inhibitor, Z-VAD-FMK, for $24 \mathrm{~h}$. Apoptotic cell death was then assessed by Annexin V/PI staining. ${ }^{* * *} \mathrm{P}<0.001$ and ${ }^{* * *} \mathrm{P}<0.0001$. LCN2, lipocalin 2; TRAIL, TNF-related apoptosis-inducing ligand.

possible role of MAPK signaling in the enhancement of the sensitivity of CRC cells to TRAIL by LCN2 silencing, LCN2 was downregulated by transfection with targeting siRNA in TRAIL-resistant cells in a time-dependent manner. The active form of MAPKs was then determined by western blot analysis with antibodies that recognize the phosphorylated forms of these kinases. As shown in Fig. 5A, only the levels of p-p38 MAPK exhibited a marked increase following the silencing of LCN2 in both cell lines.

To confirm the association between $\mathrm{p} 38$ MAPK with the alteration of TRAIL sensitivity by LCN2 regulation, the cells were pre-treated with SB203580, a p38 MAPK specific inhibitor, and apoptosis was evaluated by Annexin V/PI (Fig. 5B). The percentage of LCN2-silenced cells treated with TRAIL that underwent apoptotic death was approximately $38 \%$ in the DLD-1 cells and 27\% in the HT-29 cells. Of note, following treatment with SB203580, these percentages significantly decreased down to $22 \%$ in the DLD-1 cells and 17\% in the HT-29 cells (Fig. 5B). Western blot analysis was also performed to examine the association between $\mathrm{p} 38$ MAPK activity and LCN2 as regards
TRAIL sensitivity (Fig. 5C). The levels of phosphorylated p38 MAPK were induced by the silencing of LCN2, and they were also reduced by pre-treatment with SB203580. Conversely, downregulated LCN2 by targeted siRNA was recovered by $\mathrm{p} 38$ specific inhibitor in both cell lines. The level of DR5 was also altered by LCN2 silencing and pretreatment with SB203580. These results suggest that LCN2 is responsible for TRAIL sensitivity and is crosslinked with p38 MAPK.

CHOP, a member of the C/EBP family of transcription factors, serves as a substrate for $\mathrm{p} 38 \mathrm{MAPK}$ and is a key regulator of DR5 $(29,30)$. We thus hypothesized that CHOP is regulated by LCN2 silencing and p38 MAPK inhibition. To verify this, when LCN2 was downregulated by targeting siRNA, the level of CHOP substantially increased. Conversely, CHOP expression decreased by pre-treatment with the selective inhibitor of $\mathrm{p} 38$ MAPK in the TRAIL-resistant CRC cells (Fig. 5C). Overall, these data demonstrate that LCN2 silencing enhances TRAIL sensitivity through the regulation of p38 MAPK/CHOP/DR5 signaling and the proposed molecular mechanisms of LCN2 on TRAIL resistance are illustrated in Fig. 6. 
A

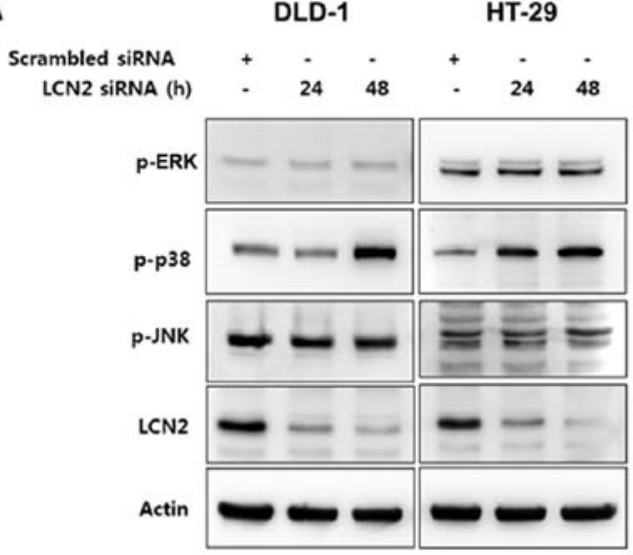

C

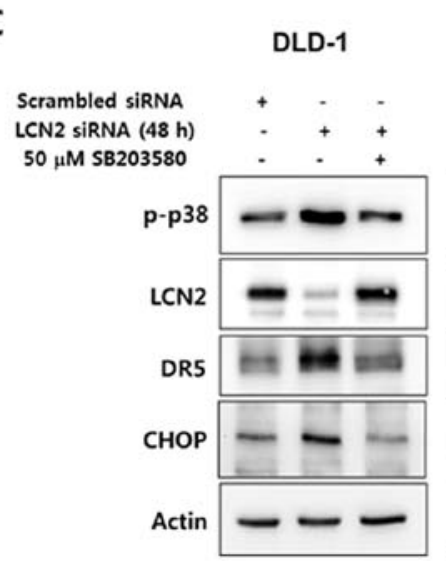

B

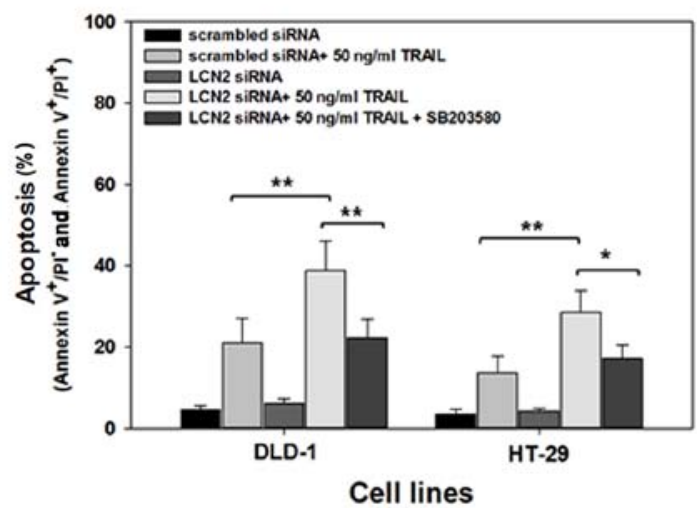

HT-29

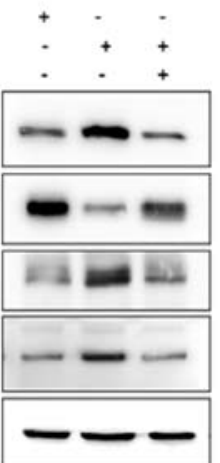

Figure 5. p38 MAPK is involved in LCN2-regulated TRAIL sensitivity in CRC cells. (A) Cells were transfected with scrambled or LCN2 siRNA for the indicated time periods and the levels of phosphorylated ERK, p38 and JNK were examined by western blot analysis. (B) Cells were transfected with LCN2 siRNA for $48 \mathrm{~h}$, and then treated with SB203580 (a specific inhibitor of p38) for $24 \mathrm{~h}$. Apoptotic cell death was assessed by flow cytometry using Annexin V/PI staining. ${ }^{*} \mathrm{P}<0.05$ and ${ }^{* *} \mathrm{P}<0.001$. (C) Transfected cells were treated with $50 \mathrm{ng} / \mathrm{ml}$ TRAIL for $24 \mathrm{~h}$ following pre-treatment with SB203580 for $2 \mathrm{~h}$. The levels of phosphorylated p38, LCN2, DR5, and CHOP were examined by western blot analysis. LCN2, lipocalin 2; TRAIL, TNF-related apoptosis-inducing ligand; CRC, colorectal cancer.

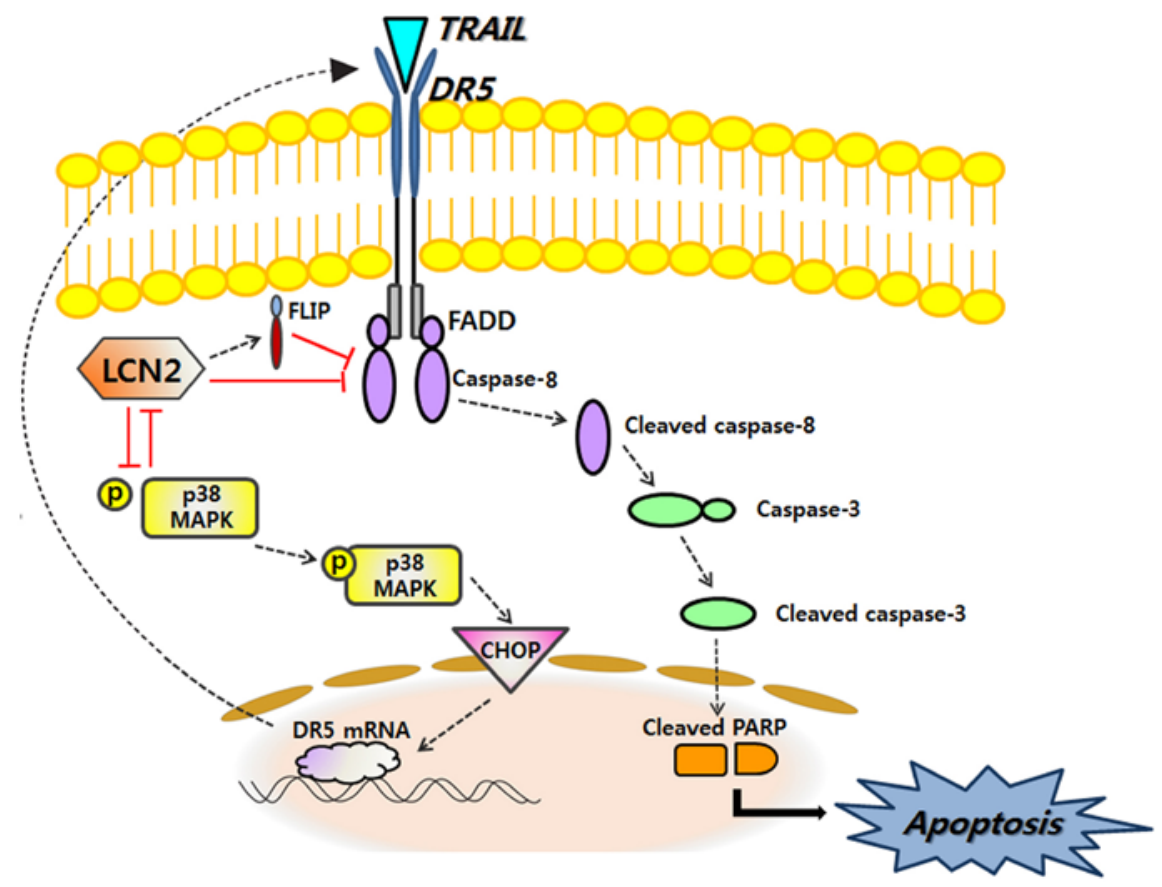

Figure 6. Schematic representation of the proposed mechanisms of LCN2 on TRAIL sensitivity and TRAIL-induced apoptosis. Black dotted lines ending with arrows and with a perpendicular red line indicate the activation and inhibition of the pathway, respectively. LCN2, lipocalin 2; TRAIL, TNF-related apoptosis-inducing ligand; FADD, Fas-associated death domain; FLIP, Fas-associated death-domain-like IL-1 $\beta$-converting enzyme (FLICE)-inhibitory protein; DR, death receptor; PARP, poly(ADP-ribose) polymerase; CHOP, C/EBP homologous protein. 


\section{Discussion}

TRAIL has been identified as a cytotoxic cancer cell specific ligand with no effect on normal cells. However, the clinical utility of TRAIL has been limited due to multiple mechanisms of TRAIL resistance (31). A substantial number of cancer cells are resistant to TRAIL, particularly in highly malignant tumors, such as pancreatic cancer, melanoma, neuroblastoma and CRC (32). Moreover, the repeated application of TRAIL to susceptible cancer cells results in the selection and expansion of TRAIL-resistant cells with acquired resistance $(33,34)$. Thus, identifying the mechanisms responsible for TRAIL resistance will not only provide insight regarding transduction of the death signal, but is also essential for designing strategies to overcome resistance to TRAIL for future clinical applications.

Several antibodies targeting DR4/5 and recombinant human TRAIL have been developed and tested for clinical use in cancer therapy. In particular, dulanermin (recombinant human TRAIL), mapatumumab (DR4 targeting monoclonal antibody), drozitumab (DR5 targeting monoclonal antibody) and conatumumab (DR5 targeting monoclonal antibody) have generally been well tolerated and appear to be safe in patients with CRC (35-38). However, the efficacy results available thus far have been disappointing. For this reason, agonists of DR4/5 have been applied for combined treatment with other chemotherapeutic agents, although these attempts have not demonstrated significantly improved efficacy over what is expected $(35,38)$. Therefore, the identification of a biomarker that can predict the sensitivity of TRAIL and increase the understanding of the TRAIL resistance mechanisms may aid in the development of targeted therapies. In this study, we provide evidence that LCN2 has potential for use as a prediction marker of TRAIL sensitivity. In addition, we demonstrate that $\mathrm{LCN} 2$ is a key mediator of resistance to TRAIL in CRC.

The expression levels of DR4 and DR5 determine the cell fate in response to TRAIL treatment. Several studies have indicated that the upregulation of DRs can sensitize cells to TRAIL-induced cell death $(26,27,39,40)$. In this study, we found that the silencing of LCN2 promoted TRAIL-induced apoptosis through the upregulation of DR5 at both the mRNA and protein level. Moreover, we demonstrated that LCN2dependent DR5 regulation contributed to the sensitizing effects of TRAIL-induced apoptosis and was an upstream event that negatively affected DR5 expression.

Numerous mechanisms have been proposed to explain the induction of DR5, including p53 induction, reactive oxygen species (ROS) regeneration and MAPK activation, which are largely dependent on cell type $(30,41)$. In particular, MAPK activation has been suggested to play a key role in DR5 induction $(42,43)$. Thus, in this study, we investigated whether MAPK plays a role in DR5 regulation by LCN2. Our findings indicated that only p38 MAPK was involved in DR5 upregulation by LCN2 silencing in TRAIL-resistant CRC cells. In addition, we provide evidence that the inhibition of p38 MAPK with the specific inhibitor, SB203580, can counteract the increased expression of LCN2, and a similar result was also observed in the analysis of apoptotic cell death using Annexin V/PI staining. These results clearly identify a high degree of crosstalk between LCN2 and p38 MAPK. Through these observations, we provide new insight into the role of LCN2 in TRAIL resistance.

$\mathrm{CHOP}$ is a $29 \mathrm{kDa}$ protein composed of an $\mathrm{N}$-terminal transcriptional activation domain and a C-terminal basicleucine zipper (bZIP) domain. In 1996, Wang and Ron demonstrated that CHOP serves as a link between a specific stress-activated protein kinase, $\mathrm{p} 38$, and cellular growth and differentiation (29). Moreover, Bruhat et al reported that p38 MAPK regulated the expression of CHOP at the post-transcriptional level (44). For that reason, CHOP expression has been considered to play a crucial role in p38 MAPK-regulated DR5 and TRAIL sensitivity. In the present study, we identified that the activation of p38 MAPK by LCN2 silencing induced CHOP expression. Our observations are supported by those of earlier study showing the role of CHOP in upregulating DR5 and enhancing the effects of TRAIL by p38 MAPK activation $(29,44)$. Inversely, the selective inhibition of p38 MAPK by SB203580 recovered the LCN2 level and abolished CHOP expression. Taken together, these results indicate that $\mathrm{CHOP}$ is regulated by $\mathrm{p} 38 \mathrm{MAPK}$ as a transcription factor. $\mathrm{CHOP}$ was also negatively regulated by LCN2 due to the crosstalk between LCN2 and p38 MAPK.

LCN2 was originally considered a biomarker of inflammation, ischemia, infection and kidney damage due to its function to capture bacterial siderophores (45). Moreover, the mRNA and protein levels of LCN2 have been shown to be elevated in various types of cancer, including CRC, demonstrating that LCN2 may serve as a cancer biomarker (46). In our previous study, we suggested the possibility of using LCN2 as a diagnostic and prognostic marker by classifying the expression pattern by CRC stage and metastasis using patient specimens (19). In addition, we identified that LCN2 negatively regulates cell proliferation and the EMT process through glycolysis using human CRC cells (19). Although the functional role of LCN2 in tumorigenesis and progression in cancer has been investigated, studies linking LCN2 to various cancer pathologies, such as chemoresistance are lacking. In this study, we confirmed that LCN2 is a key mediator of cancer resistance to TRAIL. Using human specimens and CRC cell lines, the level of LCN2 was found to be inversely associated with DR5 and cellular susceptibility to TRAIL-induced apoptosis. Furthermore, we demonstrate that LCN2-related resistance to TRAIL signaling appears to directly regulate the extrinsic apoptotic pathway and crosstalk with p38 MAPK, which is responsible for DR5-mediated apoptosis (Fig. 6). Thus, in this study, for the first time, at least to the best of our knowledge, we provide a new algorithm of LCN2 as a potential biomarker for the prediction of tumor resistance to TRAIL or the DR5 targeted therapies.

To our knowledge, this study is the first study to identify the possibility of using LCN2 as a prediction marker of TRAIL sensitivity in CRC. Using in vitro experiments, we observed that LCN2 negatively regulates p38 MAPK-mediated DR5 and mediates TRAIL-induced apoptosis via the extrinsic apoptotic pathway. Accordingly, we demonstrate a new role of LCN2 as a sensor for TRAIL response in CRC. These findings provide new insight into the role of LCN2 and the underlying molecular mechanisms of TRAIL resistance in CRC, indicating LCN2 as a potential prediction marker and therapeutic target. 


\section{Acknowledgements}

Not applicable.

\section{Funding}

This study was supported by Basic Science Research Program through the National Research Foundation of Korea (NRF) funded by the Ministry of Science, ICT and Future Planning (NRF-2015R1C1A2A01055803) and by the Fund of Biomedical Research Institute, Chonbuk National University Hospital.

\section{Availability of data and materials}

All data generated or analyzed during this study are included in this published article.

\section{Authors' contributions}

SLK and SWK conceived and planned the experiments. ISM and YRP analyzed the mRNA levels in the human CRC samples. SLK and ISM carried out all the in vitro experiments. STL and SWK contributed to the interpretation of the results. SLK and ISM took the lead in writing the manuscript. All authors provided critical feedback and helped shape the research, analysis and manuscript. All authors have read and approved the final manuscript.

\section{Ethics approval and consent to participate}

The study protocol was approved by the Institutional Review Boards of Chonbuk National University Hospital (IRB no. 2016-04-018). All patients provided written informed consent prior to collecting the tissue samples.

\section{Patient consent for publication}

Not applicable.

\section{Competing interests}

The authors declare that they have no competing interests.

\section{References}

1. Siegel RL, Miller KD and Jemal A: Cancer statistics, 2015. CA Cancer J Clin 65: 5-29, 2015.

2. Ferlay J, Soerjomataram I, Dikshit R, Eser S, Mathers C, Rebelo M, Parkin DM, Forman D and Bray F: Cancer incidence and mortality worldwide: Sources, methods and major patterns in GLOBOCAN 2012. Int J Cancer 136: E359-E386, 2015.

3. Hara K, Beppu T, Kimura M, Fujita Y, Takata T, Nishio K and Ono N: Influence of novel supramolecular substance, [2] rotaxane, on the caspase signaling pathway in melanoma and colon cancer cells in vitro. J Pharmacol Sci 122: 153-157, 2013.

4. Van Cutsem E, Köhne CH, Láng I, Folprecht G, Nowacki MP, Cascinu S, Shchepotin I, Maurel J, Cunningham D, Tejpar S, et al: Cetuximab plus irinotecan, fluorouracil, and leucovorin as first-line treatment for metastatic colorectal cancer: Updated analysis of overall survival according to tumor KRAS and BRAF mutation status. J Clin Oncol 29: 2011-2019, 2011.

5. Cheng L, Ren W, Xie L, Li M, Liu J, Hu J, Liu BR and Qian XP: Anti-EGFR MoAb treatment in colorectal cancer: Limitations, controversies, and contradictories. Cancer Chemother Pharmacol 74: 1-13, 2014
6. Hellwig CT and Rehm M: TRAIL signaling and synergy mechanisms used in TRAIL-based combination therapies. Mol Cancer Ther 11: 3-13, 2012.

7. Han B, Yao W, Oh YT, Tong JS, Li S, Deng J, Yue P, Khuri FR and Sun SY: The novel proteasome inhibitor carfilzomib activates and enhances extrinsic apoptosis involving stabilization of death receptor 5. Oncotarget 6: 17532-17542, 2015.

8. Ashkenazi A, Holland P and Eckhardt SG: Ligand-based targeting of apoptosis in cancer: The potential of recombinant human apoptosis ligand $2 /$ Tumor necrosis factor-related apoptosis-inducing ligand (rhApo2L/TRAIL). J Clin Oncol 26: 3621-3630, 2008.

9. Koehler BC, Jäger D and Schulze-Bergkamen H: Targeting cell death signaling in colorectal cancer: Current strategies and future perspectives. World J Gastroenterol 20: 1923-1934, 2014.

10. Kelley SK, Harris LA, Xie D, Deforge L, Totpal K, Bussiere J and Fox JA: Preclinical studies to predict the disposition of Apo2L/tumor necrosis factor-related apoptosis-inducing ligand in humans: Characterization of in vivo efficacy, pharmacokinetics, and safety. J Pharmacol Exp Ther 299: 31-38, 2001.

11. Kim SL, Liu YC, Park YR, Seo SY, Kim SH, Kim IH, Lee SO, Lee ST, Kim DG and Kim SW: Parthenolide enhances sensitivity of colorectal cancer cells to TRAIL by inducing death receptor 5 and promotes TRAIL-induced apoptosis. Int $\mathrm{J}$ Oncol 46: 1121-1130, 2015.

12. Skerra A: Lipocalins as a scaffold. Biochim Biophys Acta 1482: 337-350, 2000.

13. Yang J, Goetz D, Li JY, Wang W, Mori K, Setlik D, Du T, Erdjument-Bromage $\mathrm{H}$, Tempst P, Strong R, et al: An iron delivery pathway mediated by a lipocalin. Mol Cell 10: 1045-1056, 2002.

14. Devireddy LR, Gazin C, Zhu X and Green MR: A cell-surface receptor for lipocalin $24 \mathrm{p} 3$ selectively mediates apoptosis and iron uptake. Cell 123: 1293-1305, 2005.

15. Nielsen BS, Borregaard N, Bundgaard JR, Timshel S, Sehested M and Kjeldsen L: Induction of NGAL synthesis in epithelial cells of human colorectal neoplasia and inflammatory bowel diseases. Gut 38: 414-420, 1996.

16. Sanjeevani S, Pruthi S, Kalra S, Goel A and Kalra OP: Role of neutrophil gelatinase-associated lipocalin for early detection of acute kidney injury. Int J Crit Illn Inj Sci 4: 223-228, 2014.

17. Yan C, Yuanjie T, Zhengqun X, Jiayan $C$ and Kongdan L: Neutrophil gelatinase-associated lipocalin attenuates ischemia/ reperfusion injury in an in vitro model via autophagy activation. Med Sci Monit 24: 479-485, 2018.

18. Lee HJ, Lee EK, Lee KJ, Hong SW, Yoon Y and Kim JS: Ectopic expression of neutrophil gelatinase-associated lipocalin suppresses the invasion and liver metastasis of colon cancer cells. Int J Cancer 118: 2490-2497, 2006.

19. Kim SL, Lee ST, Min IS, Park YR, Lee JH, Kim DG and Kim SW: Lipocalin 2 negatively regulates cell proliferation and epithelial to mesenchymal transition through changing metabolic gene expression in colorectal cancer. Cancer Sci 108: 2176-2186, 2017.

20. Livak KJ and Schmittgen TD: Analysis of relative gene expression data using real-time quantitative PCR and the 2(-Delta Delta C(T)) Method. Methods 25: 402-408, 2001.

21. Irmler M, Thome M, Hahne M, Schneider P, Hofmann K, Steiner V, Bodmer JL, Schröter M, Burns K, Mattmann C, et al: Inhibition of death receptor signals by cellular FLIP. Nature 388: 190-195, 1997.

22. Scaffidi C, Schmitz I, Zha J, Korsmeyer SJ, Krammer PH and Peter ME: Differential modulation of apoptosis sensitivity in CD95 type I and type II cells. J Biol Chem 274: 22532-22538, 1999.

23. Nicholson DW, Ali A, Thornberry NA, Vaillancourt JP, Ding CK, Gallant M, Gareau Y, Griffin PR, Labelle M, Lazebnik YA, et al: Identification and inhibition of the ICE/CED-3 protease necessary for mammalian apoptosis. Nature 376: 37-43, 1995.

24. Tewari M, Quan LT, O'Rourke K, Desnoyers S, Zeng Z, Beidler DR, Poirier GG, Salvesen GS and Dixit VM: Yama/CPP32 beta, a mammalian homolog of CED-3, is a CrmA-inhibitable protease that cleaves the death substrate poly(ADP-ribose) polymerase. Cell 81: 801-809, 1995.

25. Kim EY, Ryu JH and Kim AK: CAPE promotes TRAIL-induced apoptosis through the upregulation of TRAIL receptors via activation of p38 and suppression of JNK in SK-Hep1 hepatocellular carcinoma cells. Int J Oncol 43: 1291-1300, 2013.

26. Lepage C, Léger DY, Bertrand J, Martin F, Beneytout JL and Liagre B: Diosgenin induces death receptor-5 through activation of p38 pathway and promotes TRAIL-induced apoptosis in colon cancer cells. Cancer Lett 301: 193-202, 2011. 
27. Ohtsuka T, Buchsbaum D, Oliver P, Makhija S, Kimberly R and Zhou T: Synergistic induction of tumor cell apoptosis by death receptor antibody and chemotherapy agent through JNK/p38 and mitochondrial death pathway. Oncogene 22: 2034-2044, 2003.

28. Pennati M, Sbarra S, De Cesare M, Lopergolo A, Locatelli SL, Campi E, Daidone MG, Carlo-Stella C, Gianni AM and Zaffaroni N: YM155 sensitizes triple-negative breast cancer to membrane-bound TRAIL through p38 MAPK- and CHOPmediated DR5 upregulation. Int J Cancer 136: 299-309, 2015.

29. Wang XZ and Ron D: Stress-induced phosphorylation and activation of the transcription factor CHOP (GADD153) by p38 MAP Kinase. Science 272: 1347-1349, 1996.

30. Yamaguchi $\mathrm{H}$ and Wang HG: CHOP is involved in endoplasmic reticulum stress-induced apoptosis by enhancing DR5 expression in human carcinoma cells. J Biol Chem 279: 45495-45502, 2004.

31. Ozören N and El-Deiry WS: Cell surface Death Receptor signaling in normal and cancer cells. Semin Cancer Biol 13: 135-147, 2003.

32. den Hollander MW, Gietema JA, de Jong S, Walenkamp AM, Reyners AK, Oldenhuis CN and de Vries EG: Translating TRAIL-receptor targeting agents to the clinic. Cancer Lett 332: 194-201, 2013.

33. Yoshida T, Zhang Y, Rivera Rosado LA and Zhang B: Repeated treatment with subtoxic doses of TRAIL induces resistance to apoptosis through its death receptors in MDA-MB-231 breast cancer cells. Mol Cancer Res 7: 1835-1844, 2009.

34. Jin Z, McDonald ER III, Dicker DT and El-Deiry WS: Deficient tumor necrosis factor-related apoptosis-inducing ligand (TRAIL) death receptor transport to the cell surface in human colon cancer cells selected for resistance to TRAIL-induced apoptosis. J Biol Chem 279: 35829-35839, 2004.

35. Wainberg ZA, Messersmith WA, Peddi PF, Kapp AV, Ashkenazi A, Royer-Joo S, Portera CC and Kozloff MF: A phase 1B study of dulanermin in combination with modified FOLFOX6 plus bevacizumab in patients with metastatic colorectal cancer. Clin Colorectal Cancer 12: 248-254, 2013.

36. Trarbach T, Moehler M, Heinemann V, Köhne CH, Przyborek M, Schulz C, Sneller V, Gallant G and Kanzler S: Phase II trial of mapatumumab, a fully human agonistic monoclonal antibody that targets and activates the tumour necrosis factor apoptosisinducing ligand receptor-1 (TRAIL-R1), in patients with refractory colorectal cancer. Br J Cancer 102: 506-512, 2010.

37. Rocha Lima CM, Bayraktar S, Flores AM, MacIntyre J, Montero A, Baranda JC, Wallmark J, Portera C, Raja R, Stern H, et al: Phase Ib study of drozitumab combined with first-line mFOLFOX6 plus bevacizumab in patients with metastatic colorectal cancer. Cancer Invest 30: 727-731, 2012.
38. Fuchs CS, Fakih M, Schwartzberg L, Cohn AL, Yee L, Dreisbach L, Kozloff MF, Hei YJ, Galimi F, Pan Y, et al: TRAIL receptor agonist conatumumab with modified FOLFOX6 plus bevacizumab for first-line treatment of metastatic colorectal cancer: A randomized phase 1b/2 trial. Cancer 119: 4290-4298, 2013.

39. Takeda K, Stagg J, Yagita H, Okumura K and Smyth MJ: Targeting death-inducing receptors in cancer therapy. Oncogene 26: 3745-3757, 2007

40. Do MT, Na M, Kim HG, Khanal T, Choi JH, Jin SW, Oh SH, Hwang IH, Chung YC, Kim HS, et al: Ilimaquinone induces death receptor expression and sensitizes human colon cancer cells to TRAIL-induced apoptosis through activation of ROS-ERK/p38 MAPK-CHOP signaling pathways. Food Chem Toxicol 71: 51-59, 2014.

41. Wu GS, Burns TF, McDonald ER III, Jiang W, Meng R, Krantz ID, Kao G, Gan DD, Zhou JY, Muschel R, et al: KILLER/DR5 is a DNA damage-inducible p53-regulated death receptor gene. Nat Genet 17: 141-143, 1997.

42. Ohtsuka T and Zhou T: Bisindolylmaleimide VIII enhances DR5-mediated apoptosis through the MKK4/JNK/p38 kinase and the mitochondrial pathways. J Biol Chem 277: 29294-29303, 2002.

43. Shenoy K, Wu Y and Pervaiz S: LY303511 enhances TRAIL sensitivity of SHEP-1 neuroblastoma cells via hydrogen peroxide-mediated mitogen-activated protein kinase activation and up-regulation of death receptors. Cancer Res 69: 1941-1950, 2009.

44. Bruhat A, Jousse C, Wang XZ, Ron D, Ferrara M and Fafournoux P: Amino acid limitation induces expression of CHOP, a CCAAT/enhancer binding protein-related gene, at both transcriptional and post-transcriptional levels. J Biol Chem 272: 17588-17593, 1997.

45. Flo TH, Smith KD, Sato S, Rodriguez DJ, Holmes MA, Strong RK, Akira $S$ and Aderem A: Lipocalin 2 mediates an innate immune response to bacterial infection by sequestrating iron. Nature 432: 917-921, 2004.

46. Candido S, Maestro R, Polesel J, Catania A, Maira F, Signorelli SS, McCubrey JA and Libra M: Roles of neutrophil gelatinase-associated lipocalin (NGAL) in human cancer. Oncotarget 5: 1576-1594, 2014. 